\title{
DEPLOYMENT OF LASER CUTTING HEAD WITH WALL CLIMBING ROBOT FOR NUCLEAR DECOMMISSIONING
}

\author{
TARIQ P. SATTAR \\ London South Bank Innovation Centre for Automation of NDT, Granta Park, Great \\ Abington, Cambridge CB21 6AL, UK \\ PAUL HILTON \\ TWI Ltd, Granta Park, Great Abington, Cambridge, CB21 6AL, UK \\ MD OMAR FARUQ HOWLADER \\ London South Bank University, 103 Borough Road, London SE10AA, UK
}

\begin{abstract}
Decommissioning of nuclear structures requires cost effective remotely operated automated cutting techniques. Cutting with solid-state lasers employing optical fibre delivery of laser power in the multi-kilowatt range and with high focus ability of the beam offers the capability of a single laser source to address more than one type of process application. The high value asset, which is the laser itself, can be situated and maintained in a safe clean area, remote from the cutting, thus allowing the system to be reused for several other decommissioning applications. The problem then is to deploy the cutting head on the remote structure. This paper describes a $5 \mathrm{~kW}$ laser cutting system deployed by a permanent magnet adhesion wall climbing robot developed to carry the payload comprising of the laser cutting head weighing $19 \mathrm{~kg}$, optical fibre cables supplying power and control signals. Steel plates up to $40 \mathrm{~mm}$ thickness can be cut at speeds of $50 \mathrm{~mm} / \mathrm{min}$ while $10 \mathrm{~mm}$ thick plates can be cut at greater speeds of 800 $\mathrm{mm} / \mathrm{min}$.
\end{abstract}

\section{Introduction}

\subsection{Laser cutting for decommissioning}

As the complexity, urgency and cost of dealing with ever increasing challenges in nuclear decommissioning increases, organisations responsible for decommissioning operations are looking for more innovative techniques to deal with the problem. What is needed is a highly automated remote technology that can deliver a noncontact and smarter dismantling process, which can cut most 
materials, produce minimum secondary waste, work at a high speed and be flexible enough to be reused in many decommissioning processes.

Laser cutting is one such technology that meets many of these requirements [1], [2] and in the past, various high power lasers have been used to demonstrate cutting of thick-section metallic materials for nuclear decommissioning applications. All lasers offer unique capabilities, but the flexibility offered by solid-state lasers, employing optical fibre delivery of the laser power, reduces complexity and risks. Development of high power disc and fibre lasers, coupled with improvements in beam delivery, and multiple channel output, have further enhanced decommissioning capability, by providing laser power in the multikilowatt regime with good focus ability of the delivered beam.

Furthermore, the high value asset, which is the laser itself, can be situated and maintained in a safe clean area, remote from the cutting, thus allowing the system to be reused for several other decommissioning applications. Current commercially available laser technology, at modest powers i.e. of the order $5 \mathrm{~kW}$, is well capable of cutting material up to $50 \mathrm{~mm}$ in thickness.

\subsection{Laser sources and equipment}

In recent years, compact high power lasers operating in the near infra-red, have become available, utilising either fibre or disc laser technology. In a fibre laser, the lasing medium is a small diameter optical fibre suitably doped, such that when pumped with diode laser power, lasing action is created in the optical fibre. The laser resonator is completed by machining diffraction gratings inside the fibre, to reflect laser light and pass some of this light out (through the same optical fibre) to form a usable beam. A typical laser module as previously described, would have a power of more than a kilowatt. To increase the available power, multiples of the units are simply bundled together using fibre splicing techniques. Using this assembly method, lasers up to 50,000W in power are now commercially available. In a disc laser, the light is generated in a thin disc of material, suitably cooled and also pumped by laser diodes. A more conventional resonator then produces the laser beam, which is focussed onto the end of the delivery optical fibre.

Fibre and disc laser technology converts electrical power to optical power three times more than carbon dioxide lasers, contain no moving parts at all, use solid state technology and as a result, are robust and compact in size. Optical fibres can be used to carry the beam to the work piece, thereby increasing the flexibility of the system and providing an opportunity for remote processing. Another important factor in laser processing is the 'beam quality' of the laser. This is a measure of how small a spot size can be formed from a given focussing lens. The fibre and disc lasers have the high beam quality necessary for laser 
cutting applications, which require high power densities on the material surface. In addition, all the technology required to switch the laser beam from one cutting head to another, down flexible but armoured optical fibres is well tested, commercially available and already in use in the highly demanding automotive manufacturing sector. Laser cutting underwater has also been demonstrated so that it has wide applicability [3].

\subsection{Remote deployment of laser head}

While TWI Ltd has developed hand held laser guns for nuclear decommissioning work to cut up metallic structures, the worker operating the laser cutter has to wear full radiation protective suits and operate in cramped and hazardous environments.

Attempts to eliminate manual cutting are being made by using snake arms that carry laser cutting tools to enter through openings and avoid obstacles by going around them. The Lasersnake2 R\&D project funded by the UK Technology Strategy Board, the Department for Energy and Climate Change, and the Nuclear Decommissioning Authority is using snake arms to deploy laser cutting heads [4].

Another possibility is to use wall climbing robots [5-7] to deploy the laser cutter. This option is used in the work reported in this paper.

\section{The wall climbing robot and design of its permanent magnet adhesion} system

To be able to survive radioactivity at nuclear decommissioning sites, a simple teleoperated differential drive wall-climbing robot was developed with the only on-board components being four DC motors (two for each side coupled with chain transmission systems), see Figure 1 . The electronic servo drives for the motors are placed in a rack outside the radiation zone away from the robot. Drive signals are transmitted via $15 \mathrm{~m}$ long cables.

The adhesion system comprises of an array of permanent Neodymium N42 magnets at an air gap distance of $15 \mathrm{~mm}$ from the surface of the steel plate on which the robot is required to climb. The magnets are placed on a $3 \mathrm{~mm}$ thick aluminium plate which acts as the floor plate of the robot. Three mild steel yokes focus the magnetic flux in the direction of the steel plate. The robot mass is $35 \mathrm{~kg}$. Figure 2 shows the climbing robot and a $5 \mathrm{~kW}$ laser cutter head developed to test cutting of a $15 \mathrm{~mm}$ thick vertical steel plate. The cutter tip




is positioned $15 \mathrm{~mm}$ above the plate surface and at an angle of $45^{\circ}$ relative to the climbing robot, see Figure 3, to allow removal of complete sections.

The additional laser cutter mass of nearly $12 \mathrm{~kg}$ and its placement at an angle of $45^{\circ}$ generates a torque that tends to make the robot slip on the surface. It was therefore important to generate sufficient frictional torque to resist this inertial torque. Experiments to obtain sufficient torque are reported in section 3.



Figure 2. Climbing robot carrying laser cutter head to cut on the right side



Figure 3. Cutting head positioned $15 \mathrm{~mm}$ above plate, $45^{\circ}$ w.r.t. robot

\section{The Laser cutting system}

In the work described here a 5kW fibre laser, manufactured by IPG Photonics, uses a 30m long, 150 micron core diameter optical fibre to deliver its power to a cutting head. The cutting head employs a $120 \mathrm{~mm}$ focal length collimating lens to render the diverging beam emerging from the end of the fibre, parallel. This beam is then diverted through 90 degrees to a focusing lens of length $250 \mathrm{~mm}$, to form a focused spot of about $0.4 \mathrm{~mm}$ in diameter.

In the laser cutting process, the interaction of the laser beam with the material being cut causes the material to heat up and melt. A cut kerf is formed by blowing away the molten material with a jet of compressed gas and then moving the cutting head. For decommissioning applications, cut quality is not really an issue so compressed air (for cheapness) is used as the assist gas.

The maximum cutting speed, for a given thickness, depends primarily on the applied laser power, the position of the laser beam focus with respect to the surface of the material and the pressure and composition of the cutting assist gas.

In addition, the tolerance to the position of the cutting nozzle tip with respect to the material surface is greatly increased, if poor cut quality can be accepted. 
Because of the high laser power and small focused spot used, the heat input to create a cut kerf is minimal. Figure 4 shows typical decommissioning cutting speeds available, as a function of material thickness, for cutting steel at $5 \mathrm{~kW}$ laser power.

Temperature measurements were made to determine how hot a test steel plate gets at a distance of $100 \mathrm{~mm}$ from the cutting point. Figures 5 and 6 show the arrangement of three temperature sensors and the line cut. Figure 7 shows the resulting temperature of a cut plate as a function of time at three points T1T3 when a vertical cut is made by the laser cutter starting at the top of a plate. Temperatures did not exceed $50^{\circ} \mathrm{C}$ at the three points indicating that the magnet system of a climbing robot placed at a distance of $100 \mathrm{~mm}$ or more from the laser cutters focus point would not be demagnetised by heat.



Figure 4. Cutting depth as a function of the cutting speed when cutting CMn steel.

\section{Design of the permanent magnet adhesion system}

The mass of the laser head fixing structure was $7.6 \mathrm{~kg}$ and the laser head was $4.06 \mathrm{~kg}$ giving a combined payload of $11.66 \mathrm{~kg}$. This payload was required to be carried by the climbing robot.

A permanent magnet adhesion system was developed with experiments conducted to optimise the adhesion force which in turn increases the anti-sliding torque.

Four configurations of N42 magnets mounted on a 3mm thick mild steel yoke were investigated plus a fifth configuration where the yoke thickness was increased to $15 \mathrm{~mm}$. Each magnet size was $50 \times 50 \times 12 \mathrm{~mm}$. The same size was used for all experiments. The five magnet configurations were all tested at a distance of $25 \mathrm{~mm}$ (air gap) from the surface of an iron plate. 


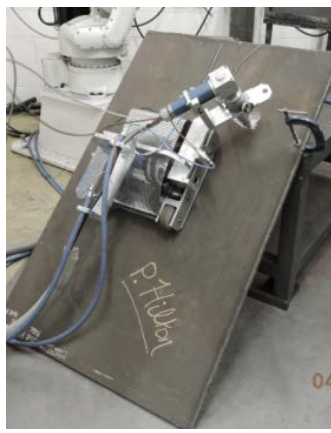

Figure 5. Measurement of temperature gradients



Figure 6. Three temperature sensors placed100mm apart and a vertical cut made



Figure 7. Temperature changes at three sensing points T1-T3 when a line cut is made at a distance of $100 \mathrm{~mm}$

The five magnet configurations are shown in Figure 8(a). Yoke dimensions are $375 \times 50 \times 3 \mathrm{~mm}$ for the first four arrangements and $375 \times 50 \times 15 \mathrm{~mm}$ for the fifth arrangement. Using thicker yokes increases the flux concentration but at the expense of greater weight.

Configuration 5 gives the best adhesion force as evidenced in Table 1 for a single yoke. In practice, the magnet system was built with three yokes placed at a distance of $30 \mathrm{~mm}$ from each other as shown in Figure 8(b). The load cell used to measure adhesion force shows a reading of $91.9 \mathrm{~kg}$ in that figure. 




Configuration 1: Yoke thickness $=3 \mathrm{~mm}$



Configuration 3: Yoke thickness $=3 \mathrm{~mm}$



Configuration 5: Yoke thickness $=15 \mathrm{~mm}$



Configuration 2: Yoke thickness $=3 \mathrm{~mm}$



Configuration 4: Yoke thickness $=3 \mathrm{~mm}$



Figure 8(b) Arrangement of three yokes, magnet configuration 5. Adhesion $92 \mathrm{~kg}$

Figure 8(a). Magnet arrangements on a single steel Yoke. Figure 8(b) Total system with 3 Yokes

Table 1. Comparison of adhesion force generated by the five magnet configurations

\begin{tabular}{ll|lll}
\hline $\begin{array}{l}\text { Magnet } \\
\text { system } \\
\text { configuration }\end{array}$ & $\begin{array}{l}\text { Yoke size } \\
\text { LxWxD } \\
\text { mm }\end{array}$ & $\begin{array}{l}\text { Total } \\
\text { system } \\
\text { mass } \\
\mathrm{kg}\end{array}$ & $\begin{array}{l}\text { Measured } \\
\text { adhesion } \\
\text { force kg }\end{array}$ & $\begin{array}{l}\text { Excess force } \\
\text { available for } \\
\text { payload kg }\end{array}$ \\
\hline 1 & $375 \times 50 \times 3$ & 2.0 & 16.0 & 14.0 \\
2 & $375 \times 50 \times 3$ & 1.23 & 13.4 & 12.17 \\
3 & $375 \times 50 \times 3$ & 1.5 & 15.0 & 13.5 \\
4 & $375 \times 50 \times 3$ & 2.0 & 22.8 & 20.8 \\
5 & $375 \times 50 \times 15$ & 3.67 & 39.4 & 35.73 \\
\hline
\end{tabular}

\section{Results with the final robot design}

The magnet system was constructed using configuration 5 repeated three times to give a measured adhesion force of $92 \mathrm{~kg}$. This adhesion force resulted in a stable robot that accurately followed vertical straight lines but drifted very slightly when moving along horizontal straight lines due to the torque generated by the laser cutter. However, for decommissioning work it is not important to cut very accurately and the present system is adequate for the purpose. 
Figure 9 captures some images from a video of the system cutting a rectangular section from the top left hand corner of the test steel plate. The robot first made a very clean vertical cut, it then rotated $90^{\circ}$ to the left to make a horizontal cut till the section was removed.


Figure 9. Removal of a rectangular section from a $15 \mathrm{~mm}$ thick steel plate

\section{References}

1. The laser alternative to nuclear decommissioning Case Study, http://www.twi-global.com/news-events/case-studies/the-laser-alternativeto-nuclear-decommissioning-479/ [Accessed 20 February 2016]

2. P. Hilton and A. Khan, Progress in the use of laser cutting for decommissioning, 2015, Industrial Laser Solutions for manufacturing, Volume 30, Issue-4

3. P. Hilton and A. Khan, New developments in laser cutting for nuclear decommissioning, WM2014 conference, March 2014

4. Laser Snake, http://www.lasersnake.co.uk/, [Accessed 20 February 2016]

5. S. Hussain, T. Sattar, E. Salinas, Parameter analysis and design framework for magnetic adhesion wall climbing wheeled robot, International Journal of Intelligent Systems Technologies and Applications (IJISTA), pp 102116, Volume 11, Nos $1 / 2$

6. J. Shang, B. Bridge, T. Sattar, S. Mondal, A. Brenner, Development of a climbing robot for the NDT of long weld lines, Industrial Robot: An international Journal, Volume 35 Issue 3, 2008

7. MD O.F. Howlader, T.P. Sattar, Development of magnetic adhesion based climbing robot for non-destructive testing," in Computer Science and Electronic Engineering Conference (CEEC), 2015, pp.105-110 